\title{
Consequence Of Anastrozole On Chemo Therapy
}

\section{Saritha Garrepalli, Stefano Cattaneo}

Department of chemotherapy.

"Corresponding Author : Saritha Garrepalli, Department of chemotherapy, Email: sarithagarrepalli@gmail.com

Received date: June 30,2017;Accepted date : August 20,2017; Published date: September 04,2017.

Citation for this Article: Saritha Garrepalli, Stefano Cattaneo. Benign Breast Lumps. J Cancer Research and Cellular Therapeutics, Doi: $10.31579 / 2640-1053 / 013$

Copyright : (c) 2017 Saritha Garrepalli . This is an open-access article distributed under the terms of The Creative Commons Attribution License, which permits unrestricted use, distribution, and reproduction in any medium, provided the original author and source are credited.

The aim of the present project work is to prepare PEGylated Anastrozole-BSA nanoparticles were prepared by desolvation technique. Prepared nanoparticles were characterized in terms of particle size, scanning electron microscope (SEM), fourier transform infrared spectroscopy (FTIR) and differential scanning calorimetry (DSC). In-vitro release studies were performed in phosphate buffer saline $\mathrm{pH} 7.4$ at $37^{\circ} \pm 0.5^{\circ} \mathrm{C}$ for 1 month. The mean particle size of obtained nanoparticles was $150-400 \mathrm{~nm}$ and was apparently spherical in shape, with smooth surface. DSC is done for the stability test for pure drug and sample.The thermogram of drug has not shifted for in the formulation compare to pure drug thermogram hence, the stability of formulation is not changed. FT-IR studies demonstrated that the drug was not changed in the formulation during the fabrication process.The encapsulation efficiency was about $48 \%$. The Anastrozole-BSA nanoparticles exhibit a most interesting release profile with small initial burst followed by slower and controlled release.

\section{Cancer}

Cancer is a term used for diseases in which abnormal cells divide without control and are able to invade other tissues. Cancer cells can spread to other parts of the body through the blood and lymph systems. The terms cancer, malignant neoplas, and malignant tumour are synonymous.

Cancer is a diseases characterised by un contrlled growth and spread of abnormal cells, which can result in death.Apoptosis is the process of programmed cel death (PCD)that may occur in multicellular organisms. Biochemical events leads to characteristic cell changes (morphology)and death. These changes include blebbing, cell shrinkage, nuclear fragmentation, chromatin condensation, and chromosomal DNA fragmentation. Generally cancer cell deviate the apoptosis process.

The national cancer act was passed in 1971. The cancer is a more than 100 different types.Mostly cancers are named for the organ or type of cell in which they start for example, cancer that begins in colon is called colon cancer ,cancer that begins in melanocytes of the skin is called melanoma.

Cancer, while the second leading cause of death in the United States, is not just one disease. It's a condition in which cells break the most fundamental rules of behavior. For any number of reasons, cancer cells fail to repair damaged DNA, they reproduce without restraint, colonize other tissues in the body and ignore signals telling them to self-destruct. The causes and consequences of cancer differ from type to type, and from patient to patient. On a molecular level, rarely are any two cancers exactly the same.

More than $50 \%$ of this cancer occur in 5major organs like lungs,colon,rectum,breast,prostate and uterus.
Cancer of lungs colon, and prostate are the principle leading causes of death in males. Where as breast and uterus cancer are most cammon in females.

\section{Background}

The word cancer is derived from the latin word. Cancer is a popular and generic word, as the actual medical name for cancer is a "neoplasia", which is derived from a greek word, which means "new formation" the oldest known description and surgical treatment of cancer was discovered in egypt and dates back to approximately 1600 B.C. Cancer is a poison that slowly spreads and concludes as contagious.

The first cause of cancer was identified by british surgeon percivall pott in 1775 . In $18^{\text {th }}$ century, it was discovered that the primary tumor spreads through the lymph nodes to other sites(metastasis).the use of surgery to treat cancer had poor results due to problems with hygiene. In $19^{\text {th }}$ century, improved surgical hygiene and surgical removal of the tumor became the primary treatment for cancer.

The age of cellular pathology was born. At the end of $19^{\text {th }}$ century, radiation therapy,non surgical cancer treatment was adopted. Since world war 2, trends in cancer treatment were to improve on a micro level the existing treatment methods, standardze them,and globalize them as a way to find cures through epidemiology and international patnerships.

This Neoplasia is a two types one is a benign neoplasm and malignant neoplasm

\section{Epidemiology}

Cancer has projected to become the leading cause of death worldwide, according to a new edition of the world cancer report from the International Agency for Research on Cancer. Cases of cancer doubled globally between 1975 and 2000, will double again by 2020, and will nearly triple by 2030, says the report. There were an estimated 12 million new cancer diagnoses and more than 7 million deaths worldwide this year. The projected numbers for 2030 are 20 to 26 million new diagnoses and 13 to 17 million deaths ${ }^{31}$.

In the developed countries cancer is the second leading cause of death accounting for $21 \%$ ( 2.5 million) of all mortality. In the developing countries cancer ranks third as a cause of death and accounts for 9.5\% (3.8 million) of all deaths. Cancer has become one of the ten leading causes of death in India ${ }^{6}$. It is estimated that there are nearly 2.5 million cancer cases at any given point of time. Over 8 lakh new cases of cancer and 4 lakh deaths occur annually due to cancer. Nearly 15 lakh patients require facilities for diagnosis, treatment and follow up at a given time ${ }^{32}$.

\section{Diagnosis}

Most cancers are initially recognized either because signs or symptoms appear or through screening. They do not give definitive diagnosis and requires the opinion of pathologist, a physician who specializes in the diagnosis of cancer and other diseases. 
People with suspected cancer are investigated with medical tests which include blood tests, X-rays, CT scans and endoscopy. The diagnosis of malignancies must be confirmed by histological examination of the cancerous cells by performing biopsy which gives information regarding the type of cell that is proliferating, its histological grade, genetic abnormalities, and other features of the tumor. cytogenetics and immunohistochemistry are other types of tests which provide information regarding molecular changes happened and also indicate the future behavior of the cancer cells ${ }^{33}$

\section{Pathophysiology}

\section{Mechanism of Metastasis:}

The mechanism of metastasis involve primary tumor, proliferation \& angiogenesis, detachment and invasion of the circulation, transport to a distant site, arrest, adherence to and extravasation through vessel wall, establishment of a microenvironment.

\section{Formation of the primary tumor:}

Tumor cells invade stroma and communicate with stromal cells. Tumor is avascular. Both tumor and host cells promote metastasis by activating growth factor pathways, allowing invasion, and promoting angiogenesis. Tumors inhibit metastasis by being antigenic and by inhibiting angiogenesis. Host cells inhibit metastasis by putting up tissue barriers, sending immune cells to kill tumor, and inhibiting angiogenesis.

\section{Progressive growth and angiogenesis :}

Angiogenesis occurs when tumors secrete angiogenic agents such as vascular endothelial growth factor (VEGF) and fibroblast growth factor (FGF) and by their recruiting lymphocytes and macrophages to secrete these agents as well. The capillary basement membrane degrades locally, creating a vascular deformity and allowing new endothelial modeling. The tumor or macrophages may also secrete anti-angiogenesis agents such as angiostatin, endostatin, and thrombospondin.

\section{Invasion :}

Tumor cells invade host stroma using enzymes, and then enter blood stream or lymphatics (which have thinner walls). The host reaction to this invasion is a fibrous ECM called the "desmoplastic response."

\section{Transport of cancer cells:}

Single cells or aggregates detach. Blood stream is very hostile to cancer cells, so aggregates survive better, and become trapped in microvasculature downstream. They adhere to endothelial cells or even the basement membrane and invade distant tissue. Growth factors supporting new cancer include transforming growth factor beta (TGF- $\beta$ ) and insulin-like growth factor-1 (IGF1). Tumor cells express chemokine receptors complementary to target organ chemokines and therefore chemokines influence patterns of spread between organs ${ }^{9}$.
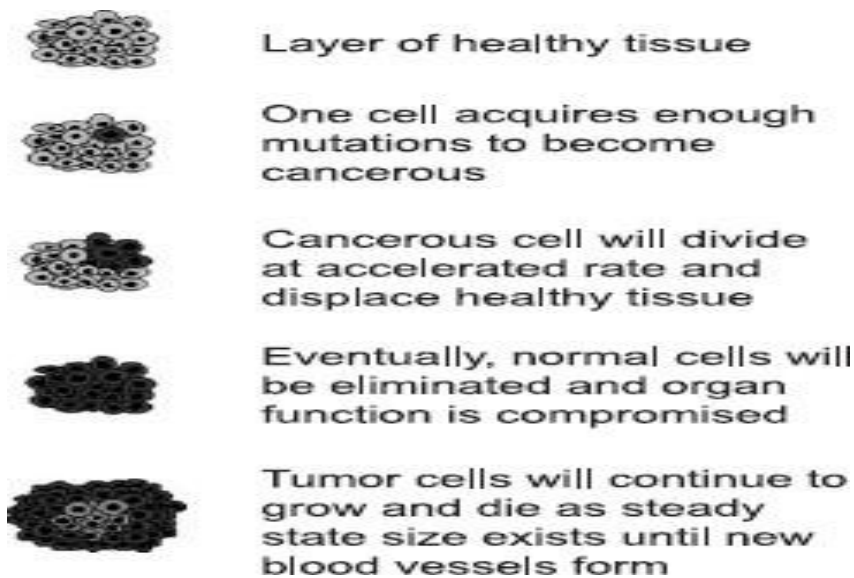

Figure.1. Tumor development from initial carcinogenesis to diffusionlimited maximal size.

\section{Causes:}

Cancer arises from one single cell. The transformation from a normal cell into a tumor cell is a multistage process, typically a progression from a pre-cancerous lesion to malignant tumors. These changes are the result of the interaction between a person's genetic factors and three categories of external agents, including:

- Physical carcinogens, such as ultraviolet and ionizing radiation.

- Chemical carcinogens, such as asbestos, components of tobacco smoke, aflatoxin (a food contaminant) and arsenic (a drinking water contaminant).

- Biological carcinogens, such as infections from certain viruses, bacteria or parasites ${ }^{10}$

\section{Clinical features-Symptoms:}

Cancer may affect people at all ages, even fetuses, but risk for the more common varieties trends to increase with age. Cancer causes about $13 \%$ of all deaths ${ }^{11}$. Roughly, cancer symptoms can be divided into three groups:

- Local symptoms: unusual lumps or swelling (tumor), hemorrhage, pain and/or ulceration.

- Symptoms of metastasis (spreading): enlarged lymph nodes, cough and hemoptysis, hepatomegaly, bone pain, fracture of affected bones and neurological symptoms. advanced cancer may cause pain.

- Systemic symptoms: Weight loss, poor appetite, fatigue and cachexia (wasting), excessive sweating (night sweats), anemia and specific paraneoplastic phenomena, i.e., specific conditions that are due to an active cancer, such as thrombosis or hormonal changes

\section{Treatmet:}

The best form of treatment will be decided based on the type of cancer and how far it has progressed in the body. A combination of treatment

options could also be used if it is believed that it will help in the treatment process. Each person undergoing treatment must have a detailed discussion with their doctor(s) about treatment options applicable to her.

Some other forms of treatment are:

\section{Hormonetherapy:}

This treatment method adds, blocks, or removes hormones. For certain conditions (such as

diabetes or menopause), hormones are given to adjust low hormone levels. To slow or stop the growth of certain cancers (such as breast cancer), synthetic hormones or other drugs may be given to block the body's natural hormones. Sometimes surgery is needed to remove the gland that makes a certain hormone. This method is sometimes also referred to as endocrine therapy.

\section{Transplantation:}

This treatment procedure restores stem cells that have been destroyed by high doses of chemotherapy and/or radiation therapy. Some common transplantation procedures are bone marrow transplantation and peripheral blood stem cell transplantationChemotherapy has become an integral component of cancer treatment for most cancers. Conventional chemotherapeutic agents still exhibit poor specificity in reaching tumor tissue and are often restricted by dose-limiting toxicity. The combination of developing controlled release technology and targeted drug delivery may provide a more efficient and less harmful solution to overcome the limitations found in conventional chemotherapy ${ }^{16}$. The efficacy of cancerous chemotherapy is often limited by serious side effects because of the toxicity of anticancer drugs to both tumor and normal cells ${ }^{38}$. 
MATERIALS

Table.1. Materials used

\begin{tabular}{|c|c|c|c|}
\hline S.No & CHEMICAL & SOURCE & PURPOSE \\
\hline $\mathbf{1}$ & Anastrozole & $\begin{array}{c}\text { Relisys } \\
\text { medical } \\
\text { devices Ltd }\end{array}$ & API \\
\hline $\mathbf{2}$ & $\begin{array}{c}\text { Bovine serum } \\
\text { albumin }\end{array}$ & SDFCL & Stabilizer \\
\hline $\mathbf{3}$ & Ethanol & SDFCL & Solvent \\
\hline $\mathbf{4}$ & PEG6000 & SDFCL & PEGylating agent \\
\hline $\mathbf{5}$ & PEG4000 & MERCK & PEGylating agent \\
\hline $\mathbf{6}$ & PEG1500 & MERCK & PEGylating agent \\
\hline $\mathbf{7}$ & Glutraldehyde & SDFCL & Cross-linking agent \\
\hline $\mathbf{8}$ & Methanol & SDFCL & Solvent \\
\hline
\end{tabular}

\section{Formulation And Experimental Methodology:}

\section{Selection of drug}

Anastrozole is used because of their anti cancer properties. Arimidex acts like an aromatase inhibitor, it aids in preventing cancer formation and development. This drug has inhibiting effects on body enzymes responsible for enzyme production. Most breast cancers require estrogen for forming and thus taking Arimidex can assist in slowing down the formation of these kinds of cancers. This reduces the chances of cancer diffusing to other body parts.

This taking Arimidex over a long period is more effective than taking tamoxifen for a similar period. In fact, this is the best hormonal therapy option for postmenopausal women suffering from early stage breast cancer. In women who normally experience recurrence, Arimidex increases the period before cancer comes back. Furthermore, it also lessens the chances of another cancer forming in the nonaffected breast.It acted through stopping the growth of the tumor (or)shrinking it.

\section{Preformulation Studies}

Preformulation is defined as phase of research and development process where physical, chemical and mechanical properties of new drug substances are characterized alone and when combined with excipient, in order to develop stable, safe and effective bioavailable dosage form.

\section{Analytical Method}

\section{Anastrozole}

$100 \mathrm{mg}$ of Anastrozole was weighed and dissolved in methanol and made up to $100 \mathrm{ml}$ with methanol in a $100 \mathrm{ml}$ volumetric flask which gives $1000 \mu \mathrm{g} / \mathrm{ml}$ of the solution. From the above solution $10 \mathrm{ml}$ of aliquot was pipetted out and made up to $100 \mathrm{ml}$ with methanol to give $100 \mu \mathrm{g} / \mathrm{ml}$ of the solution. From this solution $0.5 \mathrm{ml}$ of aliquot was pipetted out and made upto $10 \mathrm{ml}$ with methanol to give $5 \mu \mathrm{g} / \mathrm{ml}$ of the solution.

Preformulation studies for Anastrozole have been performed to know the drugs physicochemical properties so as to design it to a suitable formulation. Anastrozole is a class 2 drug classified under BCS classification with low solubility and high permeability. The physicochemical properties were described in the table 9 below.

\begin{tabular}{|c|c|}
\hline Physicochemical properties & Results \\
\hline Appearance & white in color \\
\hline Solubility & Water insoluble \\
\hline Melting point & $130.14 \mathrm{C}$ \\
\hline$\Lambda \max$ & $218 \mathrm{~nm}$ \\
\hline
\end{tabular}

Table2: Preformulation studies of Anastrozole. Auctores Publishing - Volume1-015 www.auctoresonline.org Page - 03
Poor solubility leads to poor dissolution, therefore to enhance the dissolution of the drug, different techniques have been employed such as particle size reduction by forming nanoparticles which is a novel technique.

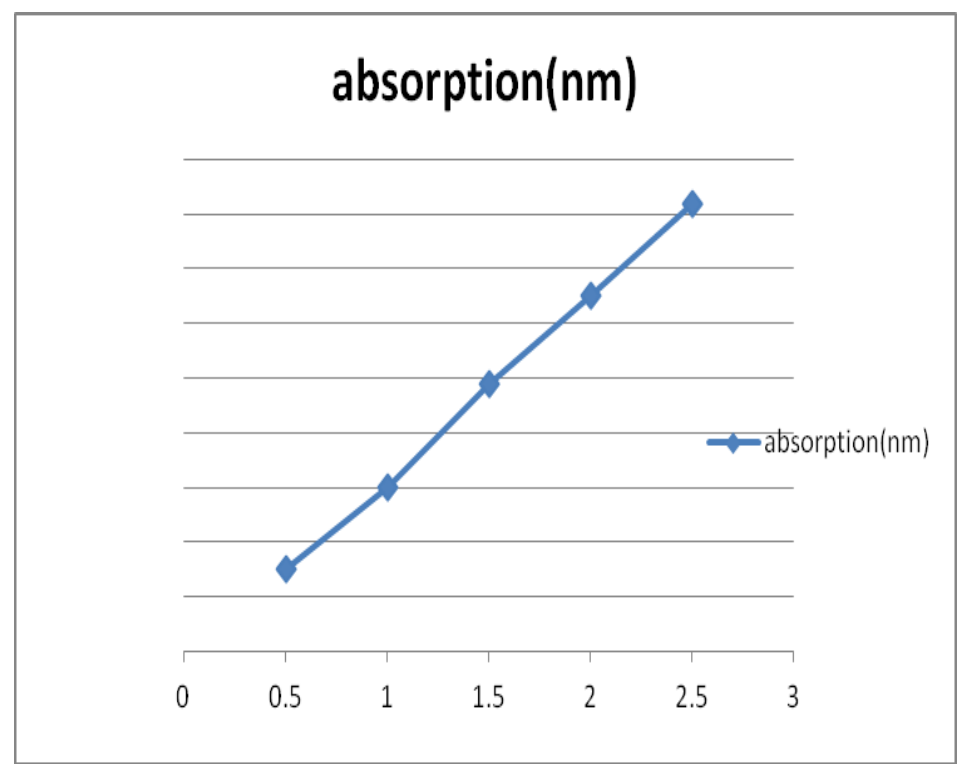

Standard calibration curve of Anastrozole

\section{Charecterization Of Nanoparticles}

\section{Particle size analysis by scanning electron microscope}

Sem was used to determine the particle size of sample Anastrozole BSA +PEG 4000 nanoparticles.the avg size of formulation was found to be in nano range of $119 \mathrm{~nm}$ to $319 \mathrm{~nm}$. Surface morphology and shape were visualized.The particles are appeared like spears. From this study it has been concluded that there is a size reduction of particle which results in enhancing dissolution rate of the anastrozole.

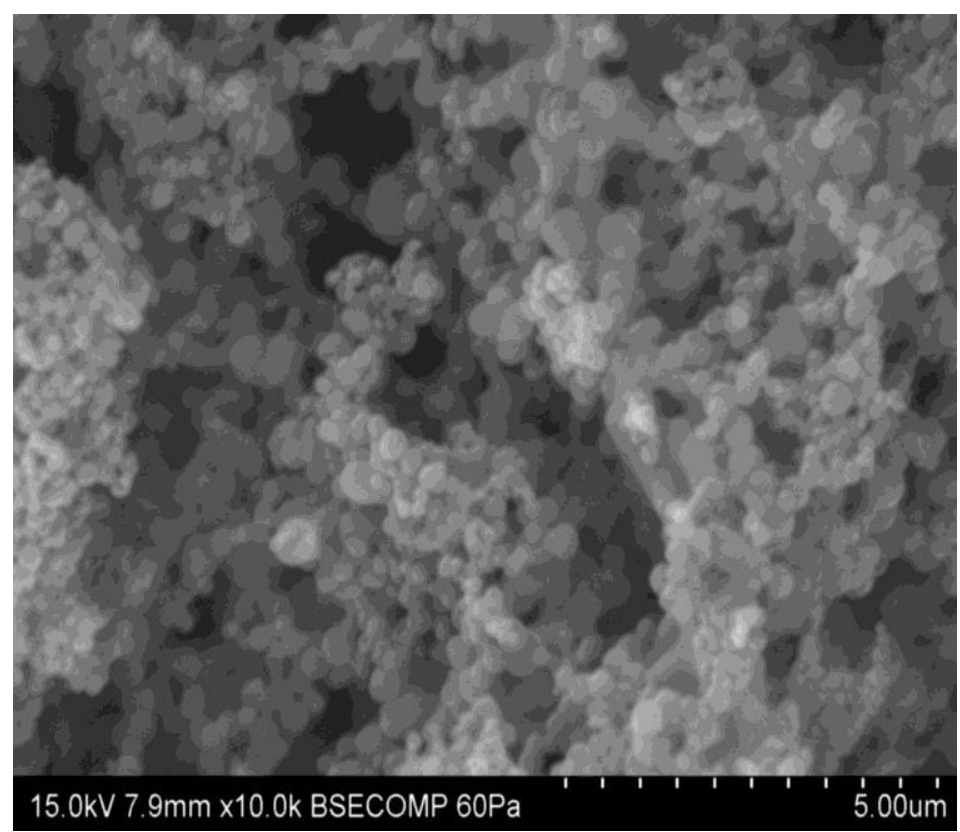

Percentage drug entrapment efficiency

\begin{tabular}{|c|c|}
\hline Formulations & \% Drug Encapsulated \\
\hline $\mathrm{F}_{1}$ & $\mathbf{3 6 \%}$ \\
\hline $\mathrm{F}_{2}$ & $\mathbf{3 9 \%}$ \\
\hline $\mathrm{F}_{3}$ & $\mathbf{4 2 \%}$ \\
\hline $\mathrm{F}_{4}$ & $\mathbf{4 8 \%}$ \\
\hline
\end{tabular}

Drug entrapment efficiency of Anastrozole nanoparticle formulations 


\section{In-vitro drug release studies}

In-vitro drug release studies of anastrozole nanoparticles were performed under magnetic stirring for a month. The calibration curve of the drug was constructed to determine the concentration of the drug from the absorbance value. From this the \% drug release was determined. The plots of cumulative percentage drug release v/s time for all the four formulations $\left(\mathrm{F}_{1}, \mathrm{~F}_{2}, \mathrm{~F}_{3}, \mathrm{~F}_{4}\right)$ were drawn and represented graphically as shown in the fig. The results of the in-vitro dissolution studies of formulation $\mathrm{F}_{1}$ to $\mathrm{F}_{4}$.

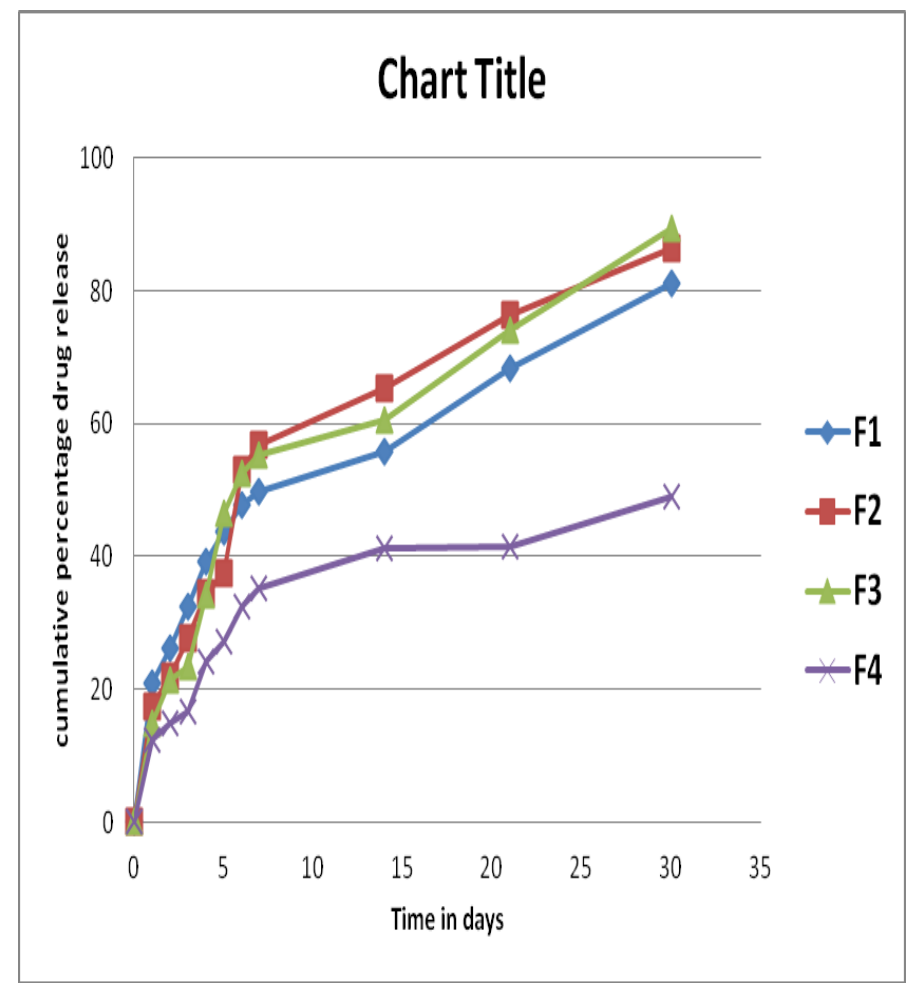

In-vitro drug release study of Anastrozole nanoparticles

\section{Conclusion}

Serum stable long circulating polymeric Anastrozole-BSA nanoparticles were successfully prepared by Desolvation technique. The control and sustained release of anastrozole nanoparticles were characterized by FT-IR, DSC and surface morphology was characterized by scanning electron microscopy. They were evaluated drug loading, percentage entrapment efficiency, particle size, in vitro release rate studies. FT-IR studies showed that similar characteristic peaks appear with out differences for the drug and their formulations; hence, it can be concluded that there was no chemical interaction between the drug and the polymer and drug was stable in the nanoparticles formulations. The DSC is done for the stability test for pure drug and formulation the thermogram of drug has not shifted for in the formulation compare to pure drug thermogram hence,the formulation stability is good. which revealed that drug is in amorphous state in the formulation. The surface morphology of Anastrozole- BSA nanoparticles was spherical in shape with smooth surface. The particles are observed aggregated might be due to the sticky nature of polymer. In particle size analysis, Anastrozole nanoparticles obtained comparatively nanosized particles range in between $150-400 \mathrm{~nm}$. The maximum drug loading and percentage entrapment efficiency was found to be $36 \%$ to $48 \%$. Thus an effective Anastrozole-BSA nanoparticle was successfully developed using a desolvation technique.

\section{References}

1. Jump up "anastrozole". Chemical Entities of Biological Interest (ChEBI). European Molecular Biology Laboratory. Retrieved 2011.
2. Jump up to: ${ }^{a b}$ Mauras N, Bishop K, Merinbaum D, Emeribe U, Agbo F, Lowe E (August 2009). "Pharmacokinetics and pharmacodynamics of anastrozole in pubertal boys with recent-onset gynecomastia". J. Clin. Endocrinol. Metab. 94 (8): 2975-8. doi:10.1210/jc.2008-2527.

3. AV Jithan, k Madhavi, M Madhavi, and K Prabhakar, International journal of pharmaceutical investigation.2011.

4. Aman U. Buzdar. Published by Elsvier. 2003, 399-403.

5. Vessela Nedelcheva Kristensen, therese Sorile, Jurgen Geisler, Noriko yoshimura, Ole- christen Linegjaerde, Ingrid Gland, Aronoldo Frigessi, Nobuhiro harada, per Eystein Lonning, Anne-Lise Borresendale. published by elsvier.2005.

6. M . Baum. European journal of cancer. 2005. 41(1667-1677)

7. Henning T.Mouridsen, Nicholas . Robert. 2005. European journal of cancer 41(1678-1689).

8. J M Nabholtz and Arimidex study group. 2003. Journal of steroid biochemistry and molecular biology 86(321-325).

9. Mamta Kalidas,Powel Brown. 2005.Clinical breast cancer, vol 6, no 1,27-37.

10. Hakki Turker Akcay, Riza Bayrak. Published by elsvier.2013.

11. Ahmad S.Zidan, Omaima A. Sammour, Mohmmad A. Hammad, Nagia A. Megrab, Muhammad D. Hussain, Mansoor A. Khan,and Muhammad J. Habib. published by elsvier. 2006.

12. Rebecca L. Glaser, Constantine Dimitrakakis. published by elsvier.2013.

13. F. Boccardo , P.Guglielmini, R. Bordonaro, A. Fini, B. Massidda M.Porpiglia, R. Roagna, P. Serra, L. Orzalesi, G.Ucci,A,Rubagotti. published by elsvier.2013.

14. Luigi F. Bertoli, James C.Barton. Remission of porphyria cutanea tarda after anastrozole treatment of breast cancer.Clinical breast cancer, vol 7,no 9, 716-718, 2007.

15. Jurgen Geisler, Ben Haynes, Dagfinn Ekse,Mitch Dowsett, Per Eystein Lonning. published by elsvier.2006.

16. Anitha K. Dunbier, Helen Anderson, Zera Ghazoui, Janine Salter, Joel S. Parker, Charles M. Perou, Ian E. Smith, Mitch Dowsett. Published by elsvier. 2011.

17. Narikazu Masuda, Yasuaki Sagara, Takayuki Kinoshitha, Hiroji Iwata, Seiga Nakamura, Yasuhiro yanagita,Reiki nishimura, Hirotaka lwase, Shunji kamigaki, Hiroyuki Takei, Shinzaburo Noguchi. published by elsvier. 2012.

18. A.Carmona-Bayonas. published by lsvier.2006.

19. Qiuling shi, Sharon H. Giordano, Huifang Lu, Angele K. Saleeba, Donna Malveaux, and Charles S.Cleeland.The journal of Pain, vol 14, no-3, 290-296,,2013.

20. I. Verote, J. Bonneterre, B. Thurlimann, J. Roobertson, M. Krzakowski, L.Mauriac, L.Koralewski, A.Webster, M.Steinberg, M.Steinberg, M.von Euler, Published by elsvier.2000.

21. Barbara. T,Mcgrogan, David Jones, Msc, and Aman B uzdar,MD. Clinical Therapeutics vol. 25,no. 11, 2007.

22. Walter Jonat, Michael gnant, Francesco Boccardo, ManfredKaufmann, Alessandra Rubagotti, Ivan Zuna, Mike GREENWOOD, Raimund Jakesz, Publised by elsvier.2006.

23. Jacksan T, Ivana Sestak, Michael Baum,Aman Buzdar, Anthony Howell, Mitch Dowsett, John F Forbes, published by elsvier. 2007.

24. J.M.Dixon, J.Jackson, M.Hills, L. Ranshaw, D.A. Cameron,T.J.Anderson,W.R.Miller, M.Dowsett, published by elsvier. 2004.

25. L.Ferreri,Fazilet Kayaselcuk,Esra Kuscu, Filiz Bolat,Hakan Sakalli, Ali Haberal. published by elsvier. 2002.

26. Vivianne Shih, Pharm D, BCOP,BCPS, Alexander Chan, PharmD, $\mathrm{MPH}, \mathrm{BCPS}, \mathrm{BCOP}$, Feng Xie, $\mathrm{PhD}$, Yu Ko, phD. published by elsvier. 2012. 